\title{
Rural public bus routing and bus stops detecting using global positioning system and geographical information system
}

\begin{abstract}
This paper reports on the investigation of rural public bus routing and passengers' boarding and alighting point detection using the application of GPS and GIS methods. Using the case study of rural bus operation in Kerian District of Perak, Malaysia, the two methods of GPS points captured during onboard survey and GIS mapping were deployed. The research aims to analyze the travel distance, travel time and passengers' boarding and alighting points of rural bus services. Bus route map was presented as the main findings of the research investigation.
\end{abstract}

Keyword: GIS mapping; GPS; Passengers' boarding and alighting; Rural bus service 